\title{
Excision-repair cross-complementing 1 predicts response to cisplatin-based neoadjuvant chemoradiotherapy in patients with esophageal squamous cell carcinoma
}

\author{
KOJI TANAKA ${ }^{1,2}$, YASUHIKO MOHRI ${ }^{1}$, MASAKI OHI ${ }^{1}$, TAKESHI YOKOE ${ }^{1}$, YUHKI KOIKE ${ }^{1}$, \\ YUHKI MORIMOTO $^{1}$, CHIKAO MIKI $^{1}$, HITOSHI TONOUCHI ${ }^{2}$ and MASATO KUSUNOKI ${ }^{1,2}$ \\ Departments of ${ }^{1}$ Gastrointestinal and Pediatric Surgery, and ${ }^{2}$ Innovative Surgery, Division of Reparative Medicine, \\ Institute of Life Sciences, Mie University Graduate School of Medicine, Mie 514-8507, Japan
}

Received November 11, 2008; Accepted May 8, 2009

DOI: 10.3892/mmr_00000190

\begin{abstract}
Low excision repair cross-complementing 1 (ERCC1) has been associated with a favorable response to cisplatin (CDDP) in several types of malignancies. The present study aimed to investigate whether ERCC1 predicts the response to CDDP-based chemoradiotherapy (CRT) in patients with esophageal squamous cell carcinoma (ESCC) and to evaluate the association between ERCC1 and platinum drug sensitivity in ESCC cell lines. ERCC1 mRNA levels in pre- and post-treatment tumoral and normal biopsies of 16 ESCC patients receiving CDDP-based CRT and in 4 ESCC cell lines were examined using real-time reverse transcription polymerase chain reaction. Pre-treatment tumoral ERCC1 was compared with clinicopathological variables and response to CRT. Responses to CDDP and oxaliplatin (OXA) in ESCC cell lines were evaluated using the WST-8 colorimetric assay by comparing ERCC1 levels. ERCC1 was significantly higher in cancer tissue compared to normal tissue $(\mathrm{p}<0.01)$. Tumoral ERCC1 significantly decreased after CRT to normal levels $(\mathrm{p}<0.05)$. ERCC1 levels in patients with a partial response were significantly lower than levels in patients who did not respond to CRT $(\mathrm{p}<0.05)$. ESCC cell lines with lower ERCC1 showed significantly greater sensitivity to clinically relevant concentrations of CDDP and OXA compared to lines with
\end{abstract}

Correspondence: Dr Koji Tanaka, Department of Gastrointestinal and Pediatric Surgery, and Department of Innovative Surgery, Division of Reparative Medicine, Institute of Life Sciences, Mie University Graduate School of Medicine, 2-174 Edobashi, Tsu, Mie 514-8507, Japan

E-mail: qouji@clin.medic.mie-u.ac.jp

Abbreviations: RT-PCR, reverse transcription-polymerase chain reaction

Key words: excision repair cross-complementing gene 1, chemoradiotherapy, cisplatin, esophageal cancer higher ERCC1 ( $\mathrm{p}<0.01)$. In conclusion, low ERCC1 levels were associated with platinum drug sensitivity in ESCC cell lines. Pre-treatment tumoral ERCC1 may be used as a predictive marker for identifying patients who respond to CRT.

\section{Introduction}

Prognosis in advanced esophageal cancer remains poor, with a 5-year survival rate of less than $20 \%$ (1). Cisplatin (CDDP) with infusional 5-fluorouracil (5-FU) and concurrent external beam radiotherapy has been adopted as the standard treatment for unresectable esophageal cancers. However, based on randomized studies, there is controversy regarding the potential benefit of the neoadjuvant treatment of resectable tumors (2-5). With a less than $30 \%$ pathologic complete response achieved with the current schedule $(6,7)$, predictive molecular markers indicating response or non-response to neoadjuvant treatment would be very helpful in selecting patients for future treatment protocols.

The excision repair cross-complementing gene 1 (ERCC1) is an excision nuclease in the nucleotide excision repair (NER) process of DNA repair pathways. In vitro and in vivo studies have shown that NER-deficient cells are hypersensitive to CDDP (8-11). ERCC1 mRNA levels have been shown to correlate with NER capacity. ERCC1 may influence the repair of platinum-DNA damage in vitro and in vivo. Various studies have evaluated the association of ERCC1 expression with response to CDDP-based chemoradiotherapy (CRT) and survival in patients with esophageal cancer, with conflicting results (12-14). This discrepancy may partly be due to the different histological types of esophageal cancer (squamous cell carcinoma and adenocarcinoma). To date, no predictive marker of the response to CDDP-based CRT is available for esophageal squamous cell carcinoma (ESCC) patients in a clinical setting.

Oxaliplatin (OXA) is a new platinum analog containing a diamino cyclohexane platinum (DACH) carrier ligand. OXA forms platinum-DNA adducts at the same nucleotide level as CDDP, distorting DNA conformation and blocking replication and transcription. Studies culturing cancer cells have demonstrated that these DACH adducts induce cell death efficiently, 
Table I. Primer and probe sequences.

\begin{tabular}{lll}
\hline Gene & Primers and probes & Sequence \\
\hline ERCC1 & Forward primer & 5'-GGGAATTTGGCGACGTAATTC-3' \\
& Reverse primer & 5'-GCGGAGGCTGAGGAACAG-3' \\
& Probe & $5^{\prime}$-CACAGGTGCTCTGGCCCAGCACATA-3' \\
$\beta$-actin & Forward primer & 5'-ACAGAGCCTCGCCTTTGC-3' \\
& Reverse primer & 5'-GCGGCGATATCATCATCC-3' \\
& Probe & 5'-CCGCCGCCAGCTCACCAT-3' \\
\hline
\end{tabular}

The primers and probe for ERCC1 were synthesized according to a previously published article.

even though OXA forms fewer DNA adducts than CDDP (15). Recently, OXA in combination with 5-FU and radiotherapy was introduced in Western countries for the treatment of patients with esophageal cancer $(16,17)$. However, it is not yet available in Japan. It is possible that a defect in NER results in hypersensitivity to OXA in ESCC, although no predictive markers for OXA sensitivity have been identified.

In this study, we examined pre-CRT ERCC1 mRNA levels in ESCC patients and assessed their value in predicting which patients would benefit from treatment with CDDP-based CRT. We also evaluated the association between ERCC1 and sensitivity to the platinum drugs CDDP and OXA in several ESCC cell lines.

\section{Materials and methods}

Patients and sample collection. The present study comprised 16 patients (15 male and 1 female, median age 65 years, range 53-77) with ESCC diagnosed between 2001 and 2006. Selection criteria included the availability of cancer and normal biopsy specimens with complete clinical data and the quality of isolated RNA for real-time PCR.

Of the 16 patients, $11(68.8 \%)$ underwent esophageal resection following chemoradiotherapy. The other 5 received definitive CRT as they refused to undergo surgery due to the risk involved in R0 resection. Endoscopic biopsy samples of the pre-treatment tumor and adjacent normal esophageal tissues were obtained from these patients. Normal esophageal mucosa was collected at a suitable distance from the primary tumor. Biopsy samples of post-treatment tumor tissues were taken from 13 patients 2 weeks after CRT. All biopsy specimens were snap frozen in liquid nitrogen and maintained at $-80^{\circ} \mathrm{C}$ until use. Prior to undergoing the pre-treatment endoscopic biopsies, all patients signed an informed consent form approving the experimental use of the tissues collected.

Cisplatin-based chemoradiotherapy regimen. Sixteen ESCC patients received systemic 5-FU and CDDP chemotherapy with concurrent radiation according to the following regimen: 4 cycles of $600 \mathrm{mg} / \mathrm{m}^{2} 5$-FU over $24 \mathrm{~h}$ by continuous intravenous administration given every week, $400 \mathrm{mg} /$ body weight UFT (Tegafur and Uracil) dispensed orally for 5 days, plus $8 \mathrm{mg} /$ day CDDP administered intravenously for 5 days concurrently with 30-40 Gy of radiation, followed by esophageal resection.
In cases of disease progression, the radiation dose was increased to a total of $60 \mathrm{~Gy}$ for definitive CRT. The time interval between neoadjuvant CRT and surgery was 2-3 weeks.

Clinical and histopathologic response to chemoradiotherapy. Tumor stage was evaluated based on the International Union Against Cancer tumor-node-metastasis (TNM) classification system (18). Clinical responses to CRT were evaluated by barium swallow, endoscopy and computed tomography. In accordance with the Guidelines for clinical and pathologic studies on carcinoma of the esophagus (1999), clinical responses were graded as complete response (CR), partial response (PR), no change (NC) or progressive disease (PD) (19).

The degree of histopathologic response was categorized as: grade 0 , neither necrosis nor regressive changes; grade $1,>2 / 3$ vital residual tumor cells (VRTCs); grade 2, <1/3 VRTCs; and grade 3, no VRTCs (19).

RNA extraction and cDNA synthesis. All samples were homogenized with a Mixer Mill MM 300 homogenizer (Qiagen Inc., Chatsworth, CA, USA). Total RNA was isolated using an RNeasy Mini Kit (Qiagen Inc.) according to the manufacturer's instructions. cDNA was synthesized with random hexamer primer and Superscript III reverse transcriptase (Invitrogen, Carlsbad, CA, USA) according to the manufacturer's instructions.

Real-time quantitative RT-PCR. Real-time quantitative RT-PCR analysis was performed using an ABI PRISM 7700 Sequence Detection System (Applied Biosystems Inc., Foster City, CA, USA). Primers and probes for $\beta$-actin, which was used as a reference gene, were designed using Primer3 software (Biology Workbench version 3.2, San Diego Supercomputer Center, University of California, San Diego, CA, USA). Primers and probes for ERCC1 were synthesized according to published sequences (12), and are shown in Table I. PCR was conducted in a final volume of $25 \mu \mathrm{l}$ with Taqman Universal PCR Master Mix (Applied Biosystems) using $0.5 \mu \mathrm{l}$ of cDNA, $900 \mathrm{nM}$ of each primer and $200 \mathrm{nM}$ of the probe for the respective genes. Cycling conditions were $50^{\circ} \mathrm{C}$ for $2 \mathrm{~min}$ and $95^{\circ} \mathrm{C}$ for $10 \mathrm{~min}$, followed by 40 cycles at $95^{\circ} \mathrm{C}$ for $15 \mathrm{sec}$ and $60^{\circ} \mathrm{C}$ for $1 \mathrm{~min}$.

Quantitation of ERCC1 mRNA expression. Relative gene expression levels were determined by the standard curve 
method. The standard curves for ERCC1 and $\beta$-actin mRNA were generated using 5-fold serially diluted solutions of cDNA from the esophageal cancer cell line TE1. All standard curves were linear in the analyzed range with an acceptable correlation coefficient $\left(\mathrm{R}^{2}\right)$. The amount of target gene expression was calculated from the standard curve, and quantitative normalization of cDNA in each sample was performed using $\beta$-actin gene expression as an internal control. Finally, ERCC1 mRNA levels were expressed as ratios to $\beta$-actin mRNA levels. Realtime PCR assays were conducted in duplicate for each sample, and the mean values were used for calculations of mRNA expression levels.

Esophageal squamous cell carcinoma lines and cell culture. The human ESCC lines TE1, TE2, KYSE70 and KYSE150 were grown in monolayer cultures in RPMI-1640 (Sigma Aldrich, St. Louis, MO, USA) supplemented with fetal bovine serum [FBS, $10 \%$ (v/v), Gibco BRL, Tokyo, Japan], glutamine (2 mM), penicillin $(100,000 \mathrm{U} / \mathrm{l})$, streptomycin $(100 \mathrm{mg} / \mathrm{l})$ and gentamycin $(40 \mathrm{mg} / \mathrm{l})$ at $37^{\circ} \mathrm{C}$ in a $5 \% \mathrm{CO}_{2}$ environment.

For routine passage, cultures were split 1:10 upon reaching $90 \%$ confluency, generally every 3 days. Cells at passages 5-9 were used for all experiments, performed with exponentially growing cells.

Anticancer agents. Cisplatin was obtained from Sigma Aldrich. Oxaliplatin was a kind gift from Yakult Co., Ltd. (Tokyo, Japan). Drugs were reconstructed in $0.9 \%$ saline for CDDP and in distilled water for OXA, and then dissolved in appropriate concentrations with distilled water and stored at $-20^{\circ} \mathrm{C}$ until use.

Growth inhibition assay. Cytotoxicity was evaluated by the WST-8 [2-(2-methoxy-4-nitrophenyl)-3-(4-nitrophenyl)-5-(2,4disulfophenyl)-2H-tetrazolium, monosodium salt] colorimetric assay. Cancer cells (5000 cells/well) were seeded into 96-well plates (Becton Dickinson Labware, NJ, USA) in $100 \mu \mathrm{l}$ of culture medium for $24 \mathrm{~h}$ prior to drug exposure.

After $24 \mathrm{~h}$ of pre-incubation, cells were treated with various concentrations of CDDP or OXA for $24 \mathrm{~h}$. Following drug exposure, the medium was discarded and replaced with $90 \mu \mathrm{l}$ of fresh medium followed by the addition of $10 \mu \mathrm{l}$ WST-8 reagent solution (Cell Counting Kit, Dojindo Laboratories, Japan) and incubated for $2 \mathrm{~h}$ at $37^{\circ} \mathrm{C}$ in an incubator.

Cell viability was determined using colorimetric comparison by reading optical density (OD) values from a microplate reader (SoftMax, Molecular Devices Corp., CA) at an absorption wavelength of $450 \mathrm{~nm}$. Cytotoxicity was evaluated by the Cell Counting kit according to the manufacturer's instructions.

Statistical analysis. All statistical analyses were carried out using JMP version 5 (SAS Institute Inc., Cary, NC, USA). For the analysis of the cell line experiment, data were represented as the mean of at least 3 independent experiments. The difference in cell viability among the 4 cell lines at each drug concentration was estimated using the Kruskal-Wallis rank sum test.

For the analysis of patient samples, results were expressed as the median value (inter-quartile range). Differences in ERCC1 levels between pre-treatment cancer and adjacent normal biopsies were evaluated using the the Mann-Whitney U test.

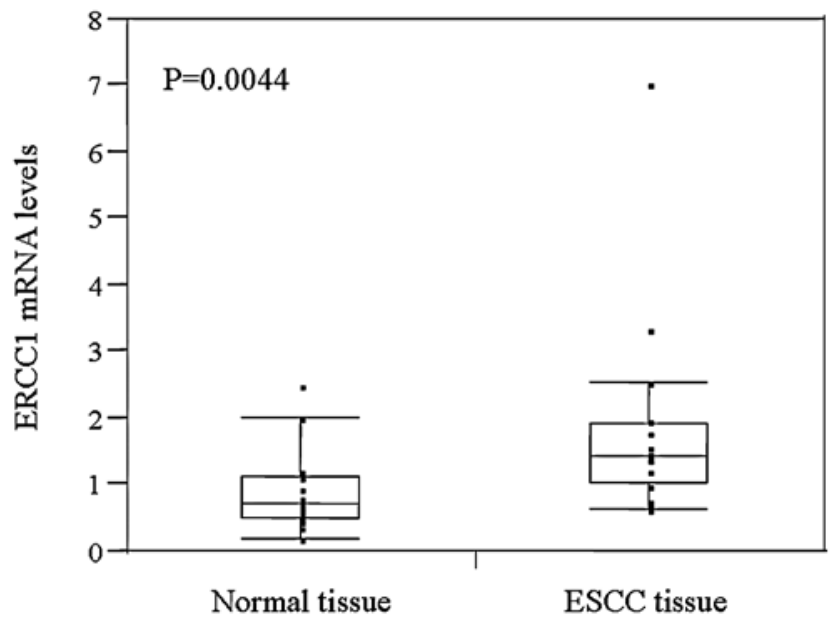

Figure 1. Pretreatment ERCC1 mRNA levels in esophageal squamous cell carcinoma (ESCC) and adjacent normal tissues. Box and whisker plots were used to summarize the distribution of mRNA levels in normal and cancer tissues. The horizontal line in the box represents the 50th (median) quartile, and the upper and lower lines of the box represent the 75th and 25th quartiles, respectively. The whiskers indicate the range of the measurements.

Associations between ERCC1 levels (continuous variables) and clinicopathological variables (categorical variables) were evaluated using the Mann-Whitney U test for two groups or the Kruskal-Wallis rank sum test for multiple groups. The paired t-test was used to compare ERCC1 levels during CRT. p-values $<0.05$ were considered statistically significant.

\section{Results}

Clinicopathological variables and ERCC1 mRNA levels. As shown in Table II, there was no statistically significant association between clinicopathological variables and ERCC1 levels. However, pre-treatment ERCC1 levels tended to be higher in older ( $>65$ years of age) patients than in younger ones $(\mathrm{p}=0.09)$.

Clinical and histopathologic response. Clinical and histopathologic responses are shown in Tables III and IV, respectively. Of the patients, $11(69 \%)$ were classified as partial response and $5(31 \%)$ as no change. According to the histopathological response criteria, $3(27 \%)$ of the 11 patients who underwent esophageal resection following CRT were categorized as grade 2 , and $8(73 \%)$ as grade 1 . No significant correlation was observed between clinical and histopathologic response.

Pre-treatment ERCC1 mRNA levels in cancer and normal tissue. ERCC1 expression values (relative mRNA levels) were expressed as the ratio between ERCC1 (the gene of interest) and $\beta$-actin (the internal reference gene). This provided a normalization factor for the amount of RNA. The median pretreatment ERCC1 mRNA level in endoscopic tumor biopsies was 0.74 (inter-quartile range $0.51-1.13$ ), whereas in adjacent normal biopsies it was 0.60 (inter-quartile range 0.43-1.50). Pre-treatment ERCC1 was significantly overexpressed in ESCC tissues compared to its expression in adjacent normal tissues ( $\mathrm{p}=0.0044)$ (Fig. 1). 
Table II. Clinicopathological characteristics and ERCC1 mRNA levels.

\begin{tabular}{|c|c|c|c|}
\hline Variable & Number & ERCC1 & P-value \\
\hline \multicolumn{4}{|l|}{ Gender } \\
\hline Male & 15 & $1.46(1.01-1.96)$ & \\
\hline Female & 1 & 1.6 (NA) & 0.59 \\
\hline Age (years) & 65 (median) & & \\
\hline$\leq 65$ & 8 & $1.30(0.82-1.71)$ & \\
\hline$>65$ & 8 & $1.54(1.47-2.40)$ & 0.09 \\
\hline Tumor size (mm) & 80 (median) & & \\
\hline$\leq 80$ & 8 & $1.46(1.21-2.55)$ & \\
\hline$>80$ & 8 & $1.47(1.10-1.77)$ & 0.91 \\
\hline \multicolumn{4}{|l|}{ Tumor location } \\
\hline Upper & 2 & $2.37(1.39-3.34)$ & \\
\hline Middle & 5 & $1.38(0.88-1.71)$ & \\
\hline Lower & 9 & $1.47(1.00-2.19)$ & 0.48 \\
\hline \multicolumn{4}{|l|}{$\mathrm{T}$ category } \\
\hline $\mathrm{T} 2$ & 3 & $1.39(0.63-1.96)$ & \\
\hline T3 & 10 & $1.54(1.11-2.75)$ & \\
\hline $\mathrm{T} 4$ & 3 & $1.38(1.01-1.47)$ & 0.52 \\
\hline \multicolumn{4}{|l|}{$\mathrm{N}$ category } \\
\hline $\mathrm{N}$ & 3 & $1.82(0.63-2.55)$ & \\
\hline No & 13 & $1.46(1.11-1.78)$ & 0.84 \\
\hline \multicolumn{4}{|l|}{ M category } \\
\hline M0 & 13 & $1.47(1.30-2.26)$ & \\
\hline Ml & 3 & $1.01(0.75-1.46)$ & 0.12 \\
\hline \multicolumn{4}{|l|}{ Clinical stage } \\
\hline Stage 2 & 5 & $1.82(1.01-2.26)$ & \\
\hline Stage 3 & 8 & $1.47(1.25-2.91)$ & \\
\hline Stage 4 & 3 & $1.01(0.75-1.46)$ & 0.30 \\
\hline
\end{tabular}

Low excision repair cross-complementing 1 (ERCC1) level values are expressed as median values (inter-quartile range). NA, not available.

Table III. Clinical response to chemoradiotherapy.

\begin{tabular}{lc}
\hline Clinical response & No. of patients $(\%)$ \\
\hline Complete response & 0 \\
Partial response & $11(69 \%)$ \\
No change & $5(31 \%)$ \\
Progressive disease & 0 \\
\hline
\end{tabular}

Change in ERCCI mRNA levels during cisplatin-based chemoradiotherapy. Of the 16 patients treated with CRT, 13 had both pre- and post-treatment tumor biopsy samples available for analysis.

ERCC1 mRNA levels were significantly lower in posttreatment tumor biopsies compared with their pre-treatment counterparts $(p=0.0103)$ (Fig. 2). The ERCC1 level in post-
Table IV. Histopathologic response to chemoradiotherapy.

\begin{tabular}{lc}
\hline Histopathologic response & No. of patients $(\%)$ \\
\hline Grade 3: No VRTCs & 0 \\
Grade 2: $<1 / 3$ VRTCs & $3(27 \%)$ \\
Grade 1: $>2 / 3$ VRTCs & $8(73 \%)$ \\
Grade 0: No regressive changes & 0
\end{tabular}

treatment tissues was downregulated in $11(85 \%)$ of the 13 ESCC patients treated with CDDP-based CRT compared with the levels found in their pre-treatment tissues.

Association of clinical and histopathologic response with ERCC1 mRNA levels. Patients with partial response (PR) to CDDP-based CRT had significantly lower ERCC1 mRNA 


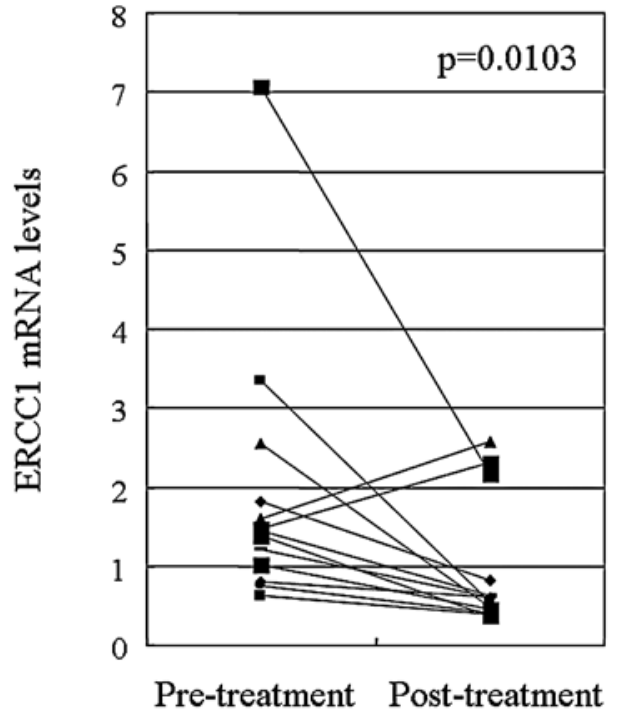

Figure 2. Pre- and post-treatment ERCC1 levels in endoscopic tumor biopsies. The pre- and post-treatment tumor biopsies of 13 patients were available. Each point indicates the serial ERCC1 level in each patient.

levels compared with those who did not respond to treatment (NC) $(\mathrm{p}=0.0107)$ (Fig. 3). However, no significant correlation was found between pre-treatment tumoral ERCC1 mRNA levels and histopathologic response (data not shown).

ERCC1 mRNA levels in esophageal cancer cell lines. To assess the relationship between ERCC1 and sensitivity to CDDP and OXA, ERCC1 mRNA levels were determined in 4 ESCC cell lines. Relative ERCC1 mRNA was 1.29 in TE1, 3.18 in TE2, 0.47 in KYSE70 and 0.47 in KYSE150. TE1 and TE2 had higher ERCC1 levels, and KYSE70 and KYSE150 had lower ERCC1 levels.

Association of ERCC1 with platinum drug sensitivity. The sensitivity of platinum drugs (CDDP and OXA) was compared in the 4 ESCC cell lines. Responses to platinum drugs showed anticancer activity in a dose-dependent manner (Fig. 4). Clinically relevant concentrations for CDDP and OXA were

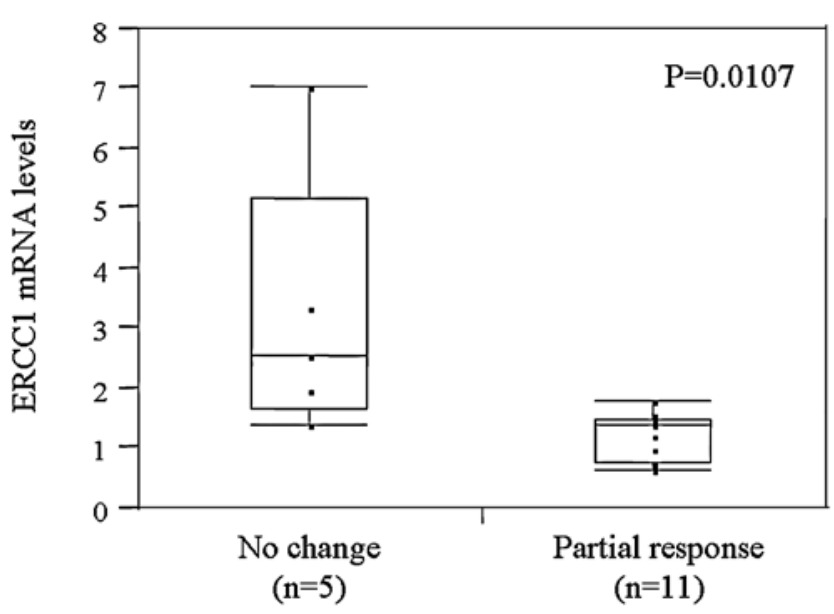

Figure 3. Association of pre-treatment ERCC1 levels in endoscopic tumor biopsy with clinical response to cisplatin-based chemoradiotherapy in ESCC patients. Box and whisker plots were used to summarize the distribution of mRNA levels in normal and cancer tissues. The horizontal line in each box represents the 50th (median) quartile, and the upper and lower lines of each box represent the 75 th and 25 th quartiles, respectively. The whiskers indicate the range of the measurements.

1-5 $\mu \mathrm{M}$ and $0.25-3 \mu \mathrm{M}$, respectively, using the plasma concentrations specified by each drug company as a reference. KYSE70 and KYSE150, with lower ERCC1, demonstrated significantly greater sensitivity to platinum drugs at clinically relevant concentrations $(1-10 \mu \mathrm{M})$ than TE1 and TE2 with higher ERCC1 (p<0.01, Fig. 5). A decreased ERCC1 level was associated with sensitivity to platinum drugs in esophageal cancer.

\section{Discussion}

In the present study, we demonstrated that patients who responded to CDDP-based CRT had significantly lower ERCC1 mRNA levels in their pre-treatment endoscopic tumor biopsies than those who did not respond to CRT. Moreoever. in an in vitro experiment using ESCC cell lines, we showed that lower levels of ERCC1 were significantly associated with greater sensitivity to platinum drugs.

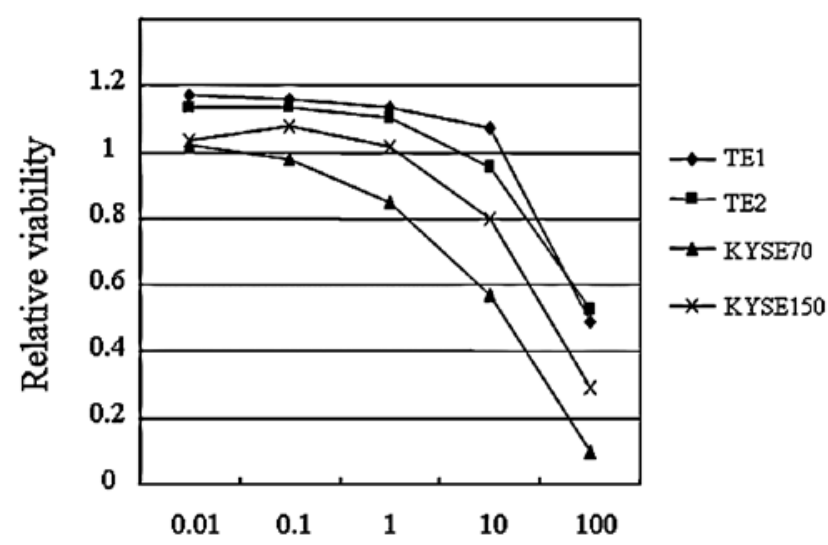

CDDP concentration ( $\mu \mathrm{M})$

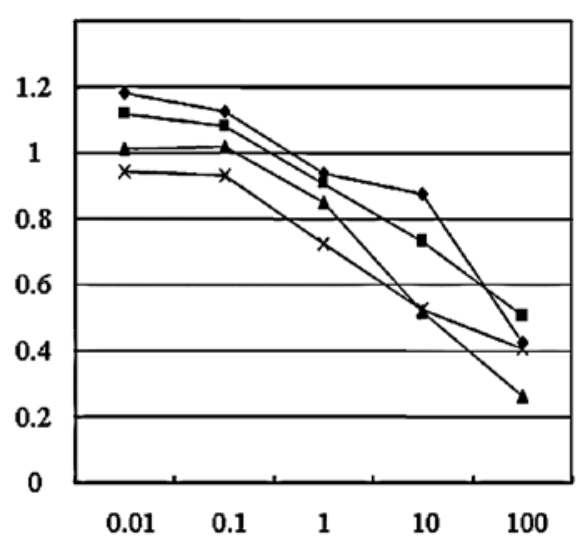

OXA concentration ( $\mu \mathrm{M})$

Figure 4. Dose response curves of cisplatin (CDDP) and oxaliplatin (OXA) in esophageal squamous cell carcinoma (ESCC) cell lines. Each relative viability value for each ESCC cell line at the indicated drug concentration represents the mean value of at least 3 independent experiments, conducted in triplicate. 

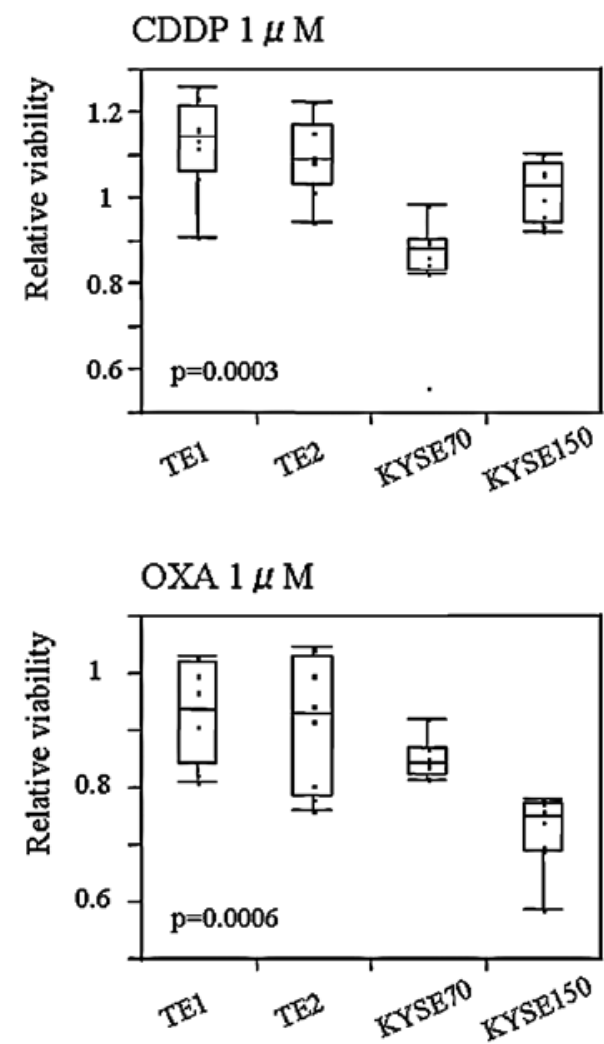

$\mathrm{CDDP} 10 \mu \mathrm{M}$

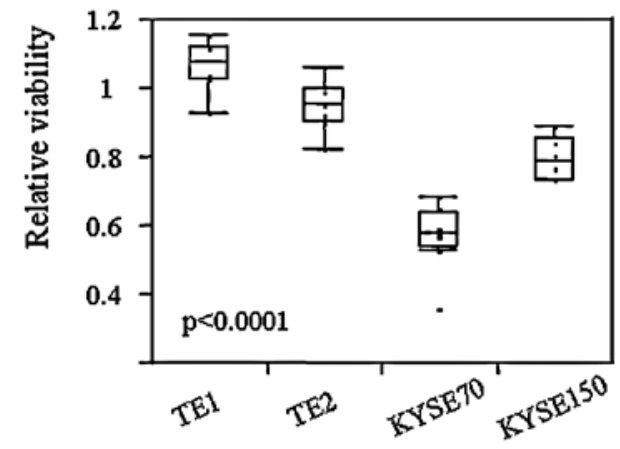

OXA $10 \mu \mathrm{M}$

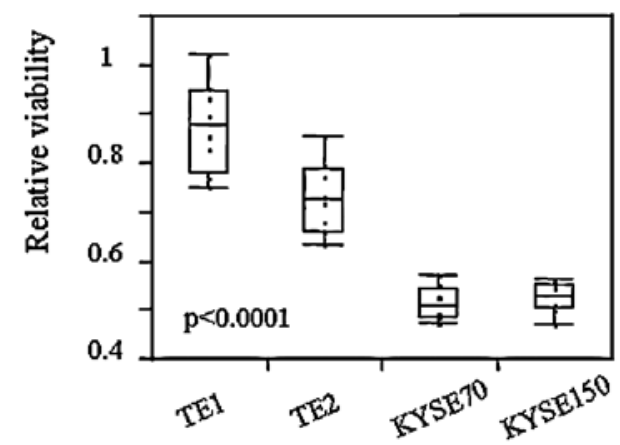

Figure 5. Growth inhibition at clinically relevant concentrations of cisplatin (CDDP) and oxaliplatin (OXA) in esophageal squamous cell carcinoma (ESCC) cell lines. Platinum drug concentrations between 1 and $10 \mu \mathrm{M}$ were considered clinically relevant concentrations of CDDP and OXA. The representative 8 values in the 4 ESCC cell lines were obtained from at least 3 independent experiments, conducted in triplicate. The difference in cell viability among the 4 cell lines at each drug concentration was estimated using the Kruskal-Wallis rank sum test. Box and whisker plots were used to summarize the distribution of relative cell viabilities in the 4 cell lines at each drug concentration. The horizontal line in each box represents the 50th (median) quartile, and the upper and lower lines of each box represent the 75 th and 25 th quartiles, respectively. The whiskers indicate the range of the measurements.

The association between ERCC1 and clinical outcome remains controversial in esophageal cancer patients treated with CDDP-based CRT followed by surgery (12-14). We showed that ERCC1 was significantly associated with clinical, but not with histopathologic, response. Although histopathologic response as opposed to clinical response appeared to be closely associated with survival, it has a critical disadvantage in that it can only be evaluated after the primary tumor has been resected. In contrast, clinical response plays an important role when tumor resectability is evaluated in locally-advanced and potentially resectable esophageal cancer. Since ERCC1 may predict clinical response to CDDP-based CRT, patient stratification and CRT regimen selection might be possible prior to pre-operative CRT.

We also showed that ERCC1 was significantly overexpressed in cancer tissue compared to normal tissue. The role of tumoral ERCC1 remains unclear $(13,20,21)$; however, it may be involved in the intrinsic DNA damage-repair ability of tumor cells, and would thus indicate the extent of accumulated intratumoral DNA damage. High tumoral ERCC1 leading to increased NER activity may be related to resistance to DNAdamaging anticancer drugs such as CDDP and OXA.

OXA is a third-generation platinum compound that has demonstrated antitumor activity against various types of malignancies, both pre-clinically (15) and clinically (16). Although OXA has not been fully evaluated in esophageal cancer, an OXA-containing regimen has demonstrated sig- nificant antitumor activity and a favorable toxicity profile in patients with metastatic esophageal cancer $(16,17)$. Our results support the idea that OXA may be clinically active in ESCC patients, and that ERCC1 may predict the response to OXA of ESCC patients.

Notably, tumoral ERCC1 was significantly decreased to normal levels following CRT. Pre-operative CRT followed by surgery has been shown to provide a pathologic complete response in $20-40 \%$ of esophageal cancer patients $(6,7)$. However, locoregional and distant recurrence have been observed even in patients with pathologic complete response. The recurrence rates of patients who did and did not undergo pre-operative CRT were similar (22). Second-line therapy is strongly recommended for these patients. However, there is no standard therapy for recurrence following CDDP-based CRT in ESCC patients. The decreased ERCC1 levels we observed after CRT indicate that platinum drugs as a second-line chemotherapy may still be appropriate for recurrent and metastatic esophageal cancer.

There are some limitations to this study, particularly in terms of the small number of clinical samples. Consequently, the results and their statistical significance should be interpreted with caution.

In conclusion, low ERCC1 expression was associated with CDDP and OXA sensitivity in ESCC cell lines. Pre-treatment tumoral ERCC1 appears to be a promising biomarker for predicting pre-operative clinical response in ESCC patients treated with CDDP-based CRT. 


\section{References}

1. Urschel JD and Vasan H: A meta-analysis of randomized controlled trials that compared neoadjuvant chemoradiation and surgery to surgery alone for resectable esophageal cancer. Am J Surg 185: 538-543, 2003.

2. Walsh TN, Noonan N, Hollywood D, Kelly A, Keeling N and Hennessy TP: A comparison of multimodal therapy and surgery for esophageal adenocarcinoma. N Engl J Med 335: 462-467, 1996.

3. Bosset JF, Gignoux M, Triboulet JP, et al: Chemoradiotherapy followed by surgery compared with surgery alone in squamouscell cancer of the esophagus. N Engl J Med 337: 161-167, 1997.

4. Urba SG, Orringer MB, Turrisi A, Iannettoni M, Forastiere A and Strawderman M: Randomized trial of preoperative chemoradiation versus surgery alone in patients with locoregional esophageal carcinoma. J Clin Oncol 19: 305-313, 2001.

5. Burmeister BH, Smithers BM, Gebski V, et al: Trans-Tasman Radiation Oncology Group; Australasian Gastro-Intestinal Trials Group: Surgery alone versus chemoradiotherapy followed by surgery for resectable cancer of the oesophagus: a randomised controlled phase III trial. Lancet Oncol 6: 659-668, 2005.

6. Chirieac LR, Swisher SG, Ajani JA, et al: Posttherapy pathologic stage predicts survival in patients with esophageal carcinoma receiving preoperative chemoradiation. Cancer 103: 1347-1355, 2005.

7. Rohatgi PR, Swisher SG, Correa AM, et al: Failure patterns correlate with the proportion of residual carcinoma after preoperative chemoradiotherapy for carcinoma of the esophagus. Cancer 104: 1349-1355, 2005.

8. Furuta T, Ueda T, Aune G, Sarasin A, Kraemer KH and Pommier Y: Transcription-coupled nucleotide excision repair as a determinant of cisplatin sensitivity of human cells. Cancer Res 62: 4899-4902, 2002.

9. Metzger R, Leichman CG, Danenberg KD, et al: ERCC1 mRNA levels complement thymidylate synthase mRNA levels in predicting response and survival for gastric cancer patients receiving combination cisplatin and fluorouracil chemotherapy. J Clin Oncol 16: 309-316, 1998.

10. Shirota Y, Stoehlmacher J, Brabender J, et al: ERCC1 and thymidylate synthase mRNA levels predict survival for colorectal cancer patients receiving combination oxaliplatin and fluorouracil chemotherapy. J Clin Oncol 19: 4298-4304, 2001.

11. Lord RV, Brabender J, Gandara D, et al: Low ERCC1 expression correlates with prolonged survival after cisplatin plus gemcitabine chemotherapy in non-small cell lung cancer. Clin Cancer Res 8 : 2286-2291, 2002.
12. Joshi MB, Shirota Y, Danenberg KD, et al: High gene expression of TS1, GSTP1, and ERCC1 are risk factors for survival in patients treated with trimodality therapy for esophageal cancer. Clin Cancer Res 11: 2215-2221, 2005.

13. Warnecke-Eberz U, Metzger R, Miyazono F, et al: High specificity of quantitative excision repair cross-complementing 1 messenger RNA expression for prediction of minor histopathological response to neoadjuvant radiochemotherapy in esophageal cancer. Clin Cancer Res 10: 3794-3799, 2004.

14. Langer R, Specht K, Becker K, et al: Association of pretherapeutic expression of chemotherapy-related genes with response to neoadjuvant chemotherapy in Barrett carcinoma. Clin Cancer Res 11: 7462-7469, 2005.

15. Raymond E, Chaney SG, Taamma A and Cvitkovic E: Oxaliplatin: a review of preclinical and clinical studies. Ann Oncol 9: 1053-1071, 1998.

16. Mauer AM, Kraut EH, Krauss SA, Ansari RH, Kasza K, Szeto L and Vokes EE: Phase II trial of oxaliplatin, leucovorin and fluorouracil in patients with advanced carcinoma of the esophagus. Ann Oncol 16: 1320-1325, 2005.

17. Jatoi A, Murphy BR, Foster NR, et al; North Central Cancer Treatment Group: Oxaliplatin and capecitabine in patients with metastatic adenocarcinoma of the esophagus, gastroesophageal junction and gastric cardia: a phase II study from the North Central Cancer Treatment Group. Ann Oncol 17: 29-34, 2006.

18. Sobin LH and Wittwkind CH: TNM Classification of Malignant Tumors, Internationale Union against Cancer. 5th edition, John Wiley \& Sons, New York, 1997.

19. Japanese Society for Esophageal Disease: Guidelines for the Clinical and Pathologic Studies on Carcinoma of the Esophagus. 9th edition, Japanese Society for Esophageal Disease, Kanehara, 1999.

20. Simon GR, Sharma S, Cantor A, Smith P and Bepler G: ERCC1 expression is a predictor of survival in resected patients with non-small cell lung cancer. Chest 127: 978-983, 2005.

21. Fautrel A, Andrieux L, Musso O, Boudjema K, Guillouzo A and Langouet S: Overexpression of the two nucleotide excision repair genes ERCC1 and XPC in human hepatocellular carcinoma. J Hepatol 43: 288-293, 2005.

22. Berger AC, Farma J, Scott WJ, et al: Complete response to neoadjuvant chemoradiotherapy in esophageal carcinoma is associated with significantly improved survival. J Clin Oncol 23: 4330-4337, 2005. 